\title{
A new device to expedite endoscopic submucosal dissection procedures: a randomized animal study of efficacy and safety (with videos)
}

Authors

Institutions
Sarah Leblanc ${ }^{1}$, Maximilien Barret ${ }^{1}$, Andreas Brehm² ${ }^{2}$, Alexandre Rouquette ${ }^{3}$, Marine Camus ${ }^{1}$, Erich Wintermantel ${ }^{2}$, Frederic Prat ${ }^{1}$

${ }^{1}$ Gastroenterology Unit, Cochin Hospital, Assistance Publique-Hôpitaux de Paris, Université Paris Descartes, Paris, France ${ }^{2}$ Institute of Medical and Polymer Engineering, Technische Universität München, Munich, Germany

3 Pathology Department, Cochin Hospital, Assistance Publique-Hôpitaux de Paris, Université Paris Descartes, Paris, France
Bibliography

DOI http://dx.doi.org/

10.1055/s-0034-1392216

Published online: 15.9.2015

Endosc Int Open 2015; 03:

E443-E449

(c) Georg Thieme Verlag KG

Stuttgart · New York

E-ISSN 2196-9736

Corresponding author

Frederic Prat, MD, PhD

Gastroenterology and

Endoscopy Unit

Cochin Hospital

Assistance Publique-Hôpitaux

de Paris

Université Paris Descartes

Sorbonne Paris Cité

75014 Paris

France

Fax: +33-158411965

frederic.prat@cch.aphp.fr
Background and study aims: Endoscopic submucosal dissection (ESD) is a recognized method for the curative treatment of superficial neoplasia, but its use is limited by lengthy procedures and the lack of versatility of existing knives. We developed a prototype ESD device with the ability to work as a needle, hook, or "scythe." This new device was compared to regular ESD knives in a randomized animal study.

Patients and methods: Eight pigs underwent two gastric ESD procedures each, similar in size and difficulty, one with a regular ESD device and the other with the new device. The order and location of each ESD, as well as the performing operator, were randomized. Primary judgment criterion was safety of procedures. Overall and submucosal dissection procedure times were measured. Time-to-surface ratios were measured and esti-

\section{Introduction}

\section{$\nabla$}

Endoscopic Submucosal Dissection (ESD) is a recently introduced technique to achieve en-bloc resections of digestive epithelial or subepithelial tumors, sparing the muscularis propria (MP). ESD was described and developed in the early 2000 s by Japanese authors and has been widely accepted in Far Eastern countries as a carcinologic method of treating superficial tumors with good prognostic features [1 - 3]. A quick Medline search yields more than 1200 publications up to the end of 2012, including thousands of patients and some randomized studies, showing that ESD is safe, reliable, and effective. However, despite its clear advantages - a minimally invasive approach allowing en-bloc resections with clean deep and lateral margins for histopathologic analysis - the spread of ESD in western countries has been much slower and remains restricted to a small number of skilled endoscopists in a few referral centers $[4,5]$. Such a disappointing outcome more than 10 years after the introduction of the mated for ESDs larger than those performed. Histopathology of the resected tissue and remaining stomach was done after each experiment.

Results: No complications were observed throughout the study and all resections were completed en-bloc and uneventfully. The submucosal extension of resections was similar with both the standard and the new devices. A comparison of time-consumption between groups did not show statistically significant differences, but a dramatic reduction of procedure duration was observed in some procedures with the new device; based on observed data, a potential timesaving of up to $66 \%$ was anticipated, with a relatively short learning curve.

Conclusions: This new versatile device proved to be as safe as regular ESD knives, and seems likely to help reduce the duration of the procedure.

technique is probably due to some well-known shortcomings of ESD, such as a long and demanding training curve, a very high time consumption relative to the amount of resected tissue, as well as the lack of versatility of many ESD devices [4, 6]. Because the development of ESD is desirable, given the increasing incidence of early/superficial carcinomas resulting from widespread cancer screening and improved diagnostic techniques, it seems necessary to make the procedure simultaneously technically easier, sufficiently versatile to be used in the most difficult anatomical conditions, less time-consuming, and more affordable [7]. Our aim was to develop a new device that would fulfill those objectives and this is the first assessment of such a device in a porcine model. 


\section{Patients and methods}

$\nabla$

\section{ESD device}

The device was designed in partnership with Medwork Medical Products and Services GmbH (Höchstadt an der Aisch, Germany) as a monopolar electrosurgical knife to be used by deploying the active wire out of the catheter sheath via a handle equipped with an electric connection port, as with most existing ESD knives. The major difference with this device is that the instrument can be used as a needle when only the very tip of the wire is deployed ( $2 \mathrm{~mm}$ in the current prototype) and also like a "scythe" when the wire is fully deployed. The needle position is typically suited to a peripheral incision, as with any other ESD device, whereas the "scythe" position offers a much longer electrical contact surface, which can make progression during the submucosal phase of the ESD procedure much easier and faster. In the "scythe" position, the $0.4-\mathrm{mm}$ wire can be oriented easily over $360^{\circ}$ by rotating the handle, so that the cutting plane remains always strictly parallel to the submucosal plane. When deployed halfway, the wire presents in the form of a hook, and can be used as such, to grab and cut fibers in the submucosa. $\bullet$ Fig. 1 shows these different configurations.

Furthermore, the prototype used was designed with additional features, including a slightly enlarged tip that improves the safety of the incision when the catheter is placed bluntly on the muscular plane, and a flushing port that makes use of the same lumen as the cutting wire for removal of coagulation debris from the wire, cleaning of the operating field, and inflation of the submucosa during ESD. Pressure at the catheter outlet, however, is not sufficient to lift the mucosa during the early phase of ESD (i.e., before mucosal incision) unlike the high pressure achieved with devices such as the Erbejet ${ }^{\mathrm{TM}}$-Hybrid-knife ${ }^{\mathrm{TM}}$ system [9]. Therefore, an injection needle is mandatory for performing ESD with this instrument and the flushing port can be used for the same purpose as a Flush-knife ${ }^{\mathrm{TM}}$. Fig. 2 shows the prototype. - Video 1 show how to maneuver it.

\section{Animals}

Eight 35-kg male or female pigs from the same farm, were used for the study. The pigs were housed at our facility during the 48 hours before the procedure. Endoscopies were performed under general anesthesia. All animals were prepared for anesthesia with a 12hour diet, and received intramuscular injection of $10 \mathrm{mg} / \mathrm{kg}$ ketamine and $2 \mathrm{mg} / \mathrm{kg}$ azaperone (Stresnil ${ }^{\circledR}$, Janssen Cilag, Titusville, New Jersey) 30 minutes before induction. After induction with $8 \mathrm{mg} / \mathrm{kg}$ of intravenous (IV) propofol (1\% Diprivan ${ }^{\circledR}$, AstraZeneca, London, UK) and endotracheal intubation, ventilation was performed with a Siemens $900 \mathrm{C}$ ventilator (Siemens, Solna, Sweden). Anesthesia was maintained with inhaled $1 \%$ to $2 \%$ isoflurane (Abbott, Abott Park, IL). All animals receivedd IV infusion of $10 \mathrm{mg} / \mathrm{kg} / \mathrm{h}$ cristalloid solution. The experimental protocol received approval from the scientific committee of the Surgical School of Paris (Ecole de Chirurgie de l'Assistance Publique - Hôpitaux de Paris, Paris, France). Experiments were performed according to the standard guidelines of the French Ministry of Agriculture, which regulates animal research in France.

\section{Study design}

The study was designed as a small-sample randomized trial whose main aim was to compare the efficacy, ease of use, and safety of the new device $v s$ a high-quality conventional ESD knife. The conventional or "regular" ESD knife used was the 2-mm straight (I-type) Flush-Knife ${ }^{\mathrm{TM}}$ (Fujifilm Corporation). The two endoscopists performing ESDs (FP, SL) each had experience with 100 or more ESDs in patients at the time the study started and more than 1 year of experience with the Flush-knife ${ }^{\mathrm{TM}}$. Their only experience with the new device was in use of the first prototype devices on a few cases in live pigs a few weeks before randomization of animals for this study.

\section{ESD description and group allocation}

Each animal in the study underwent two gastric ESDs in the same locations, one in the anterior wall approximately $4 \mathrm{~cm}$ below the cardia and close to the lesser curvature, and the other one next to the first, also on the anterior wall but closer to the greater curvature and a bit more distant in the stomach. All procedures were performed in the forward position and none in retroflexion. These locations were chosen for their ease of reproduction from one animal to the next one. All ESDs were approximately the same size, between 3.5 and $4 \mathrm{~cm}$ "Lesion" diameter was measured with the help of an adult biopsy forceps with the jaws open, which was moved four times along a straight line in both horizontal and vertical axes. After marking four quadrants with a biopsy, a circular marking was done around these four points by coagulation dots. This method allowed a fairly reproducible "lesion" size.

In all animals, one ESD was performed with a "regular" ESD device (Flush-knife ${ }^{\mathrm{TM}}$ ), and the other one was performed with the new device. The order in which both ESDs were done was randomized. All procedures were performed by the same senior and ESD-trained endoscopists (FP and SL); in each animal, one ESD was done by FP and the other one by SL; at the end of each series, the same number of ESDs had been done by both endoscopists with each of the devices to be compared (regular and new), and the same number of ESDs had been done with each of the devices in both gastric locations, so as to eliminate both operator and anatomical biases.

The ESD technique was conventional, with peripheral marking using the device (regular or new), submucosal lifting with indigo-stained $9 \%$ saline, peripheral incision with a 1- to 2-mm margin beyond markings, and submucosal dissection with the help of a transparent distal attachment (model D-201-10704, Olympus corp, Tokyo, Japan). After completion of each ESD, the resected specimen was fixed on cork for histopathology. All procedures were achieved with a Storz gastroscope (Karl Storz GmH, Tuttlingen, Germany) and an ERBE ICC 350 electrosurgical unit (ERBE, Tubingen, Germany). Electrical settings were Endocut and Forced Coagulation $40 \mathrm{~W}$.

The animal was sacrificed with $100 \mathrm{mg} / \mathrm{kg}$ IV injection of $7 \mathrm{mg}$ of pentobarbital (Dolethal ${ }^{\circledR}$, Vétoquinol, Paris, France) after completion of both ESDs. A gastrectomy was done and the stomach was opened to check for any accidental perforation unrecognized during procedures, lesions of the serosal face of the stomach, and accurate measurement of the mucosal defects. After macroscopic examination and measurement with a calliper, specimens were fixed for 24 hours in $10 \%$ buffered formalin, embedded in paraffin, and processed into 5 - $\mu \mathrm{m}$ sections. For histological examination, specimens were stained with Hematoxylin and Eosin and examined by a pathologist (AR). All procedures were videorecorded and the overall procedure duration was measured from insertion to final removal of the endoscope. Video clips of the main steps of a gastric ESD are presented along with this article ( Videos 2-4). Descriptive statistics were used. Quantitative 

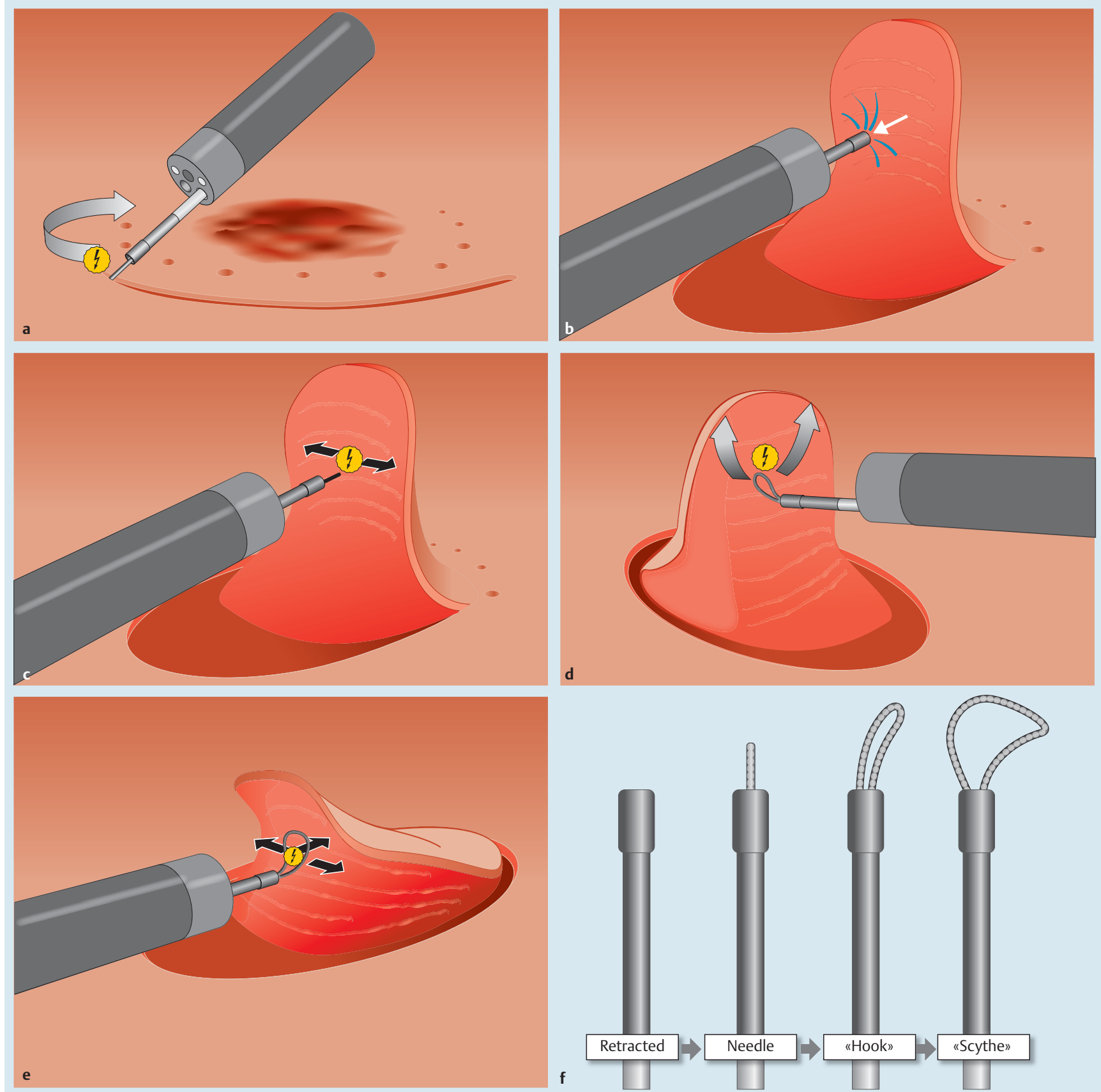

Fig. 1 The new ESD device: how it works (drawings). a "Needle" position: marking and incision. b Submucosal flushing and lifting. c "Needle" position for point-by-point submucosal dissection. $\mathbf{d}$ "Hook" position for submucosal dissection. e "Scythe" position for submucosal dissection. $\mathbf{f}$ The 4 different positions of the device.

data were compared using the paired Student's t-test for small samples.

\section{Judgment criteria}

Our primary judgment criterion was safety, as assessed by the rate of complications (essentially perforations) that occurred during the procedures or observed at necropsy. Another important outcome measure was efficacy, measured by the time needed to complete procedures. Because the statistically significant demonstration of a moderate improvement in procedure time would require a large number of experiments and animals, we did not tailor the sample to accommodate this criterion. However, procedure duration was analyzed by subdividing time into

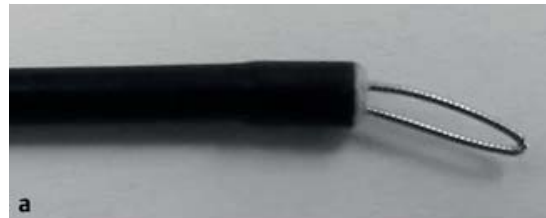

Fig. 2 The new ESD device (photos). a Catheter tip, opened halfway. b Catheter tip, fully opened. 


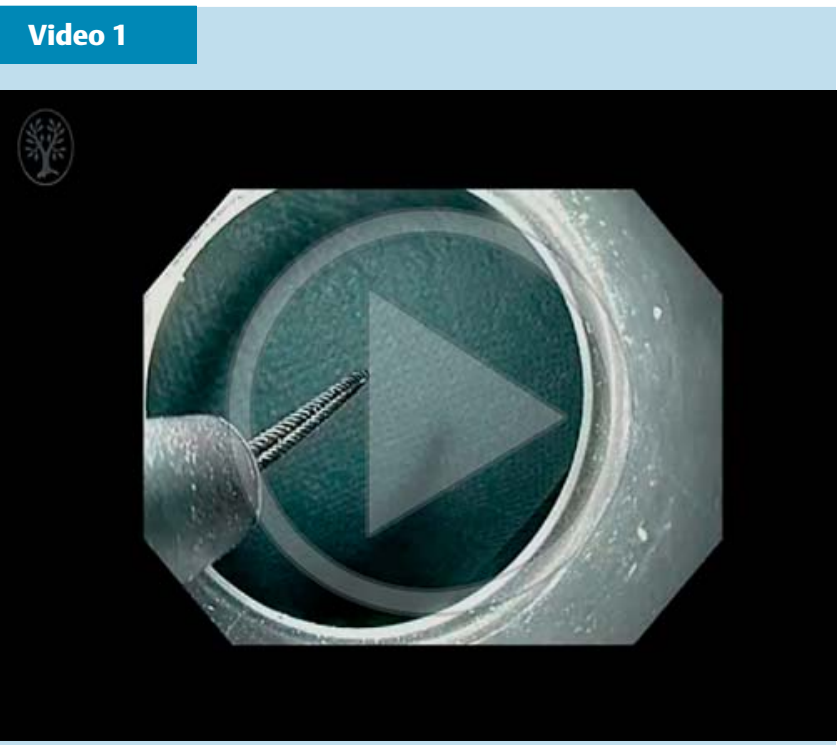

ESD device deployment and rotation. Online content including video sequences viewable at: http://dx.doi.org/10.1055/s-0034-1392216

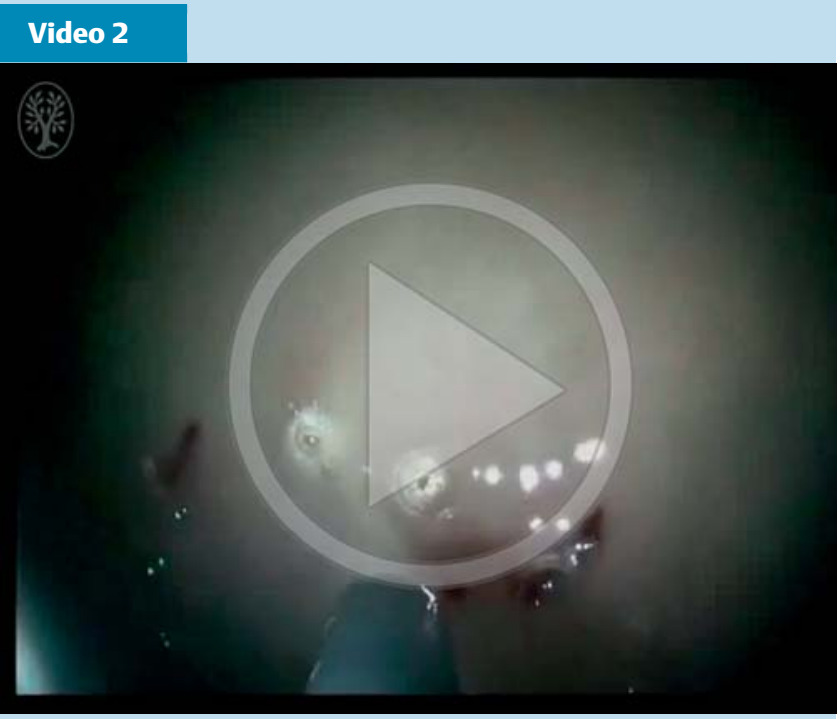

Marking ESD lesion and peripheral incision with the new ESD device. Online content including video sequences viewable at: http://dx.doi.org/10.1055/ s-0034-1392216

duration of circumferential incision and duration of submucosal dissection. We estimated a potential time saving as a function of the lesion area, based on observed minimum and maximum procedure time/area ratios with both devices. Because the dimensions of resected tissue could differ slightly from one animal to another as well as within each experiment, a ratio of time to area was calculated for each ESD. Accessory criteria were the tissue and knife visibility and the ease of use of the device, as perceived by both endoscopists.

\section{Video 3}

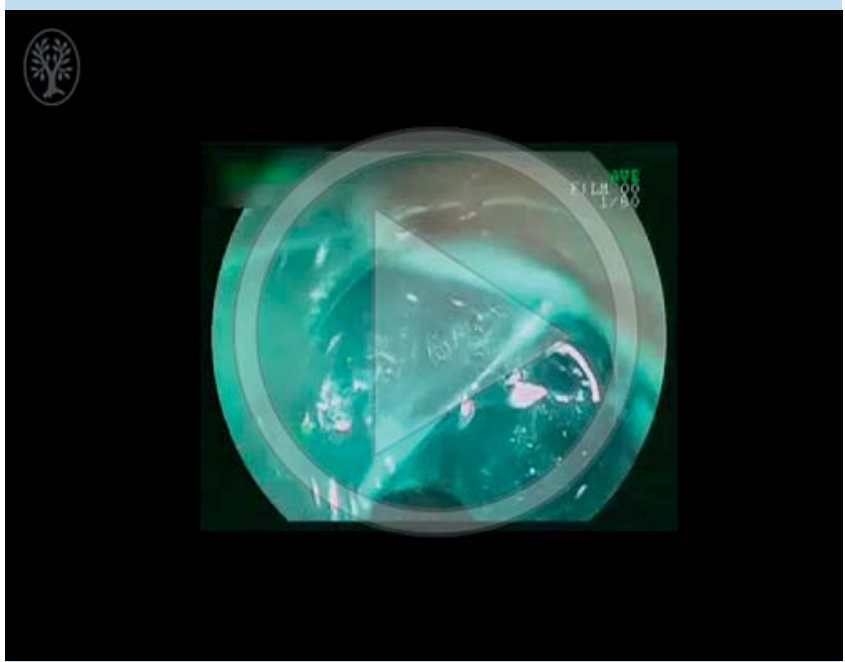

Submucosal dissection with the new ESD device in the "needle" and "hook" position. Online content including video sequences viewable at: http://dx. doi.org/10.1055/s-0034-1392216

\section{Video 4}

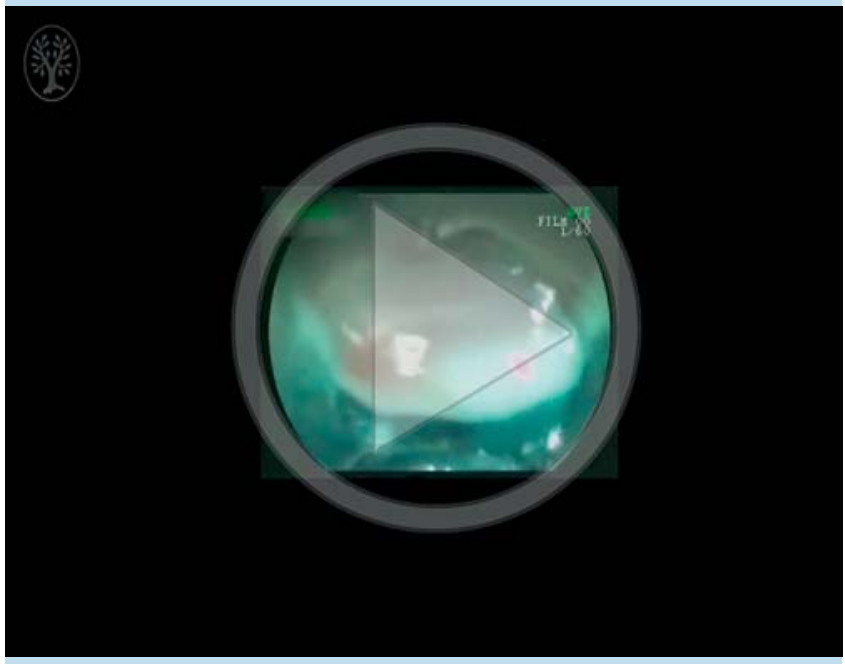

Submucosal dissection with the new ESD device in the "scythe" position. Online content including video sequences viewable at: http://dx.doi.org/ 10.1055/s-0034-1392216

\section{Results}

$\nabla$

ESD procedures were carried out uneventfully in all cases. In particular, no perforation was observed both during procedures and after autopsy on macroscopic examination of the gastric wall in the two groups. The visibility of catheter tip was excellent in all cases, as was that of the needle tip.Switching from one shape to another (i.e., hook to scythe to needle and back to hook) and fine-tuning the orientation of the wire (like "scything" parallel to the MP or "hooking" away from MP) were feasible in all cases without any dysfunction. Flushing through the lumen was found to be efficient in cleaning the operating field, but not always enough in lifting the submucosa.

All three available knife shapes (needle, hook, scythe) were used in all procedures with the new device. The scythe shape was 
Table 1 Characteristics of gastric ESDs

\begin{tabular}{|c|c|c|c|c|c|c|c|}
\hline $\mathbf{N}^{\circ}$ animal & Type of ESD device ${ }^{1}$ & $\begin{array}{l}\text { Circular } \\
\text { Incision Time } \\
\text { (min) }\end{array}$ & $\begin{array}{l}\text { Submucosal } \\
\text { Dissection Time } \\
\text { (min) }\end{array}$ & $\begin{array}{l}\text { Overall Dissec- } \\
\text { tion Time } \\
\text { (min) }\end{array}$ & $\begin{array}{l}\text { Lesion Diameter } \\
(\mathrm{Max} \times \mathrm{min} \text {, } \\
\mathrm{mm})\end{array}$ & $\begin{array}{l}\text { Lesion Area } \\
\left(\mathrm{mm}^{2}\right)\end{array}$ & $\begin{array}{l}\text { Time to Sur- } \\
\text { face Ratio } \\
\left(\mathrm{min} / \mathrm{cm}^{2}\right)\end{array}$ \\
\hline \multirow[t]{2}{*}{1} & Regular & - & - & 42 & $35 \times 20$ & 593.65 & 7.07 \\
\hline & New knife & - & - & 26 & $28 \times 20$ & 452.16 & 5.75 \\
\hline \multirow[t]{2}{*}{2} & Prototype & - & - & 40 & $37 \times 30$ & 880.9 & 4.54 \\
\hline & New knife & - & - & 35 & $45 \times 25$ & 961.6 & 3.64 \\
\hline \multirow[t]{2}{*}{3} & Regular & - & - & 50 & $28 \times 22$ & 490.62 & 10.18 \\
\hline & New knife & - & - & 50 & $30 \times 25$ & 593.65 & 8.41 \\
\hline \multirow[t]{2}{*}{4} & Regular & - & - & 36 & $30 \times 20$ & 490.62 & 7.33 \\
\hline & New knife & - & - & 36 & $30 \times 20$ & 490.62 & 7.33 \\
\hline \multirow[t]{2}{*}{5} & Prototype & 3 & 25 & 28 & $30 \times 20$ & 490.62 & 5.70 \\
\hline & New knife & 5 & 23 & 28 & $30 \times 20$ & 490.62 & 5.70 \\
\hline \multirow[t]{2}{*}{6} & Regular & 6 & 31 & 37 & $36 \times 25$ & 730.24 & 5.07 \\
\hline & New knife & 4 & 15 & 19 & $43 \times 35$ & 1193.98 & 1.60 \\
\hline \multirow[t]{2}{*}{7} & Prototype & 5 & 11 & 16 & $38 \times 34$ & 1017.36 & 1.57 \\
\hline & New knife & 10 & 21 & 31 & $35 \times 30$ & 829.15 & 3.74 \\
\hline \multirow[t]{2}{*}{8} & Regular & 7 & 18 & 25 & $28 \times 28$ & 615.44 & 4.07 \\
\hline & New knife & 4 & 18 & 22 & $28 \times 26$ & 572.26 & 3.84 \\
\hline
\end{tabular}

${ }^{1}$ Regular device corresponding to Flush-Knife ${ }^{\mathrm{TM}}$ ( $\left.2 \mathrm{~mm}\right)$.

${ }^{2}$ Lesion area is approximated as a disc, using the formula $3.14 \times([\text { Diam Max + Diam min }] / 2 / 2)^{2}$

mostly used during the median or central part of the ESD, when the submucosal plane offered full and large visibility and a feeling of safety, whereas the needle shape was used essentially for edge incision and the hook shape for initial and final submucosal cuttings. Macroscopic findings are summarized in $\bullet$ Table 1. The lesions were similar in size in the two groups $\left(8.97 \mathrm{~cm}^{2}\right.$ in the "regular" knife group and $8.76 \mathrm{~cm}^{2}$ in the "new knife" group, respectively $P=0.85$ ). Microscopically, histopathologic analysis of resected tissue and gastric wall after ESD revealed similar features with both the regular and the new devices. Most of the submucosa was resected (approximately $500 \mu \mathrm{m}$ ) with the mucosa and muscularis mucosae, whereas only a tiny fraction of the submucosa remained attached to the muscularis propria ( $\bullet$ Fig. $\mathbf{3})$. Although microscopic measurements of incision depth or thickness were not done on all resected specimens, no difference was observed between the thickness of submucosa after dissection with the regular and with the new devices. No undesirable contacts of the wire tip with the mucosa during "scythe" resection were observed macroscopically or microscopically in resected tissues.

No significant differences in time needed to complete the procedures were observed between procedures with the new devices vs the regular one (mean ESD time of $29.6 \pm 11.56$ minutes for the new device vs $35.5 \pm 7.95$ minutes for the regular one, $P=0.11$ ), but that was as expected, given the small numbers of animals involved and procedures performed. However, overall procedure times were never longer with the new device than with the regular one; on the contrary, procedure time with the new device was dramatically shorter in several procedures ( $\bullet$ Table $\mathbf{1}$ ). Findings were the same when overall ESD procedure time was calculated as a fraction of the lesion area, approximated as a disc with a trend to shorter procedures with the new device (a mean of $3.80 \mathrm{~min} / \mathrm{cm}^{2}$ for the new device $v \mathrm{~s} 4.33 \mathrm{~min} / \mathrm{cm}^{2}$ for the regular one), but not statistically different $(P=0.22)$. Some procedures were remarkably shorter with the new device, particularly in the latest experiments ( $\boldsymbol{O}$ Fig. $\mathbf{4 a}$ ). In addition, when the submucosal phase of the dissection was taken into account separately, as was done with the last four animals, the same trend was observed, with two experiments showing no or only a little time

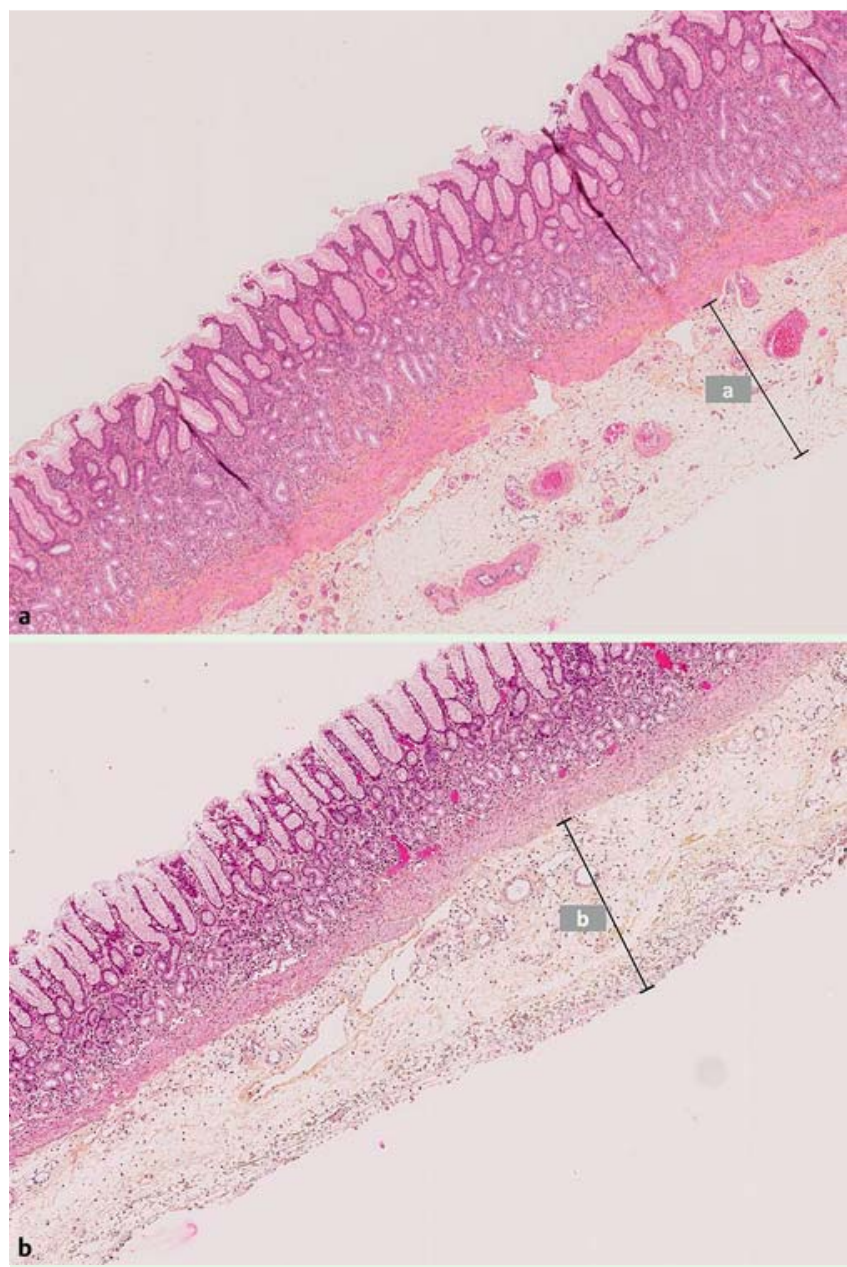

Fig. 3 Histopathology of gastric ESDs specimen, with thickness of the submucosa resected (respectively a and b), $-\mathrm{HE}$, X5. a ESD performed with the conventional device (a: $495 \mu \mathrm{m})$. b ESD performed with the new device (b: $535 \mu \mathrm{m})$. 


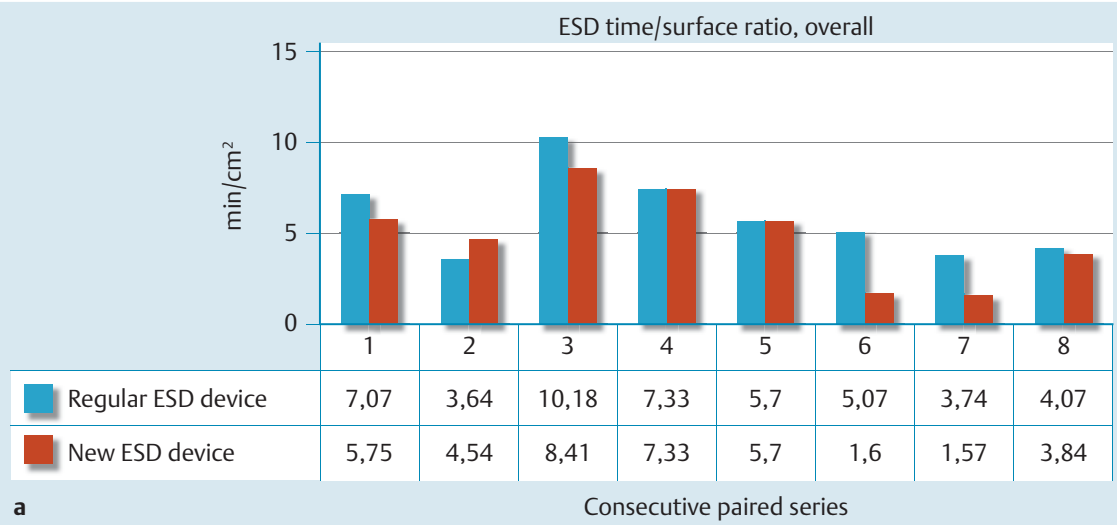

ESD time/surface ratio, submucosal

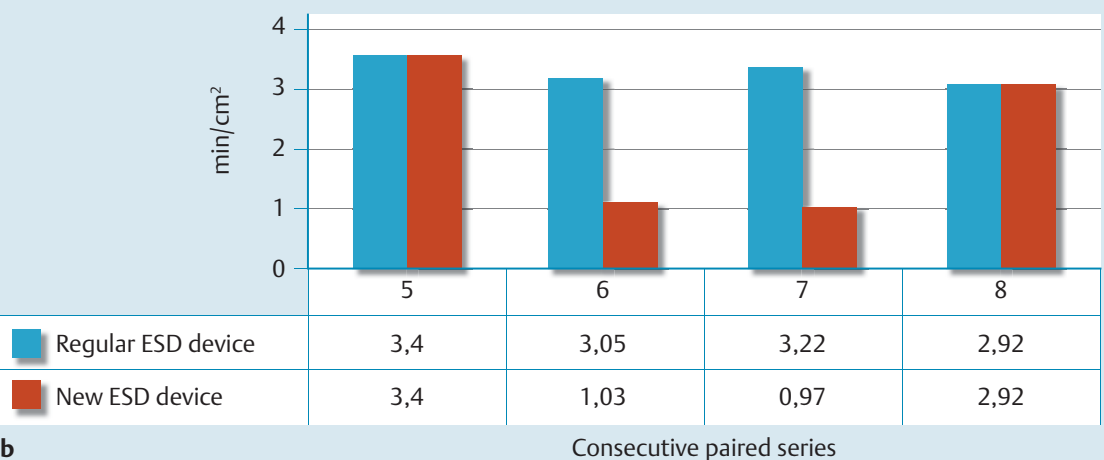

Estimated ESD time/area relation

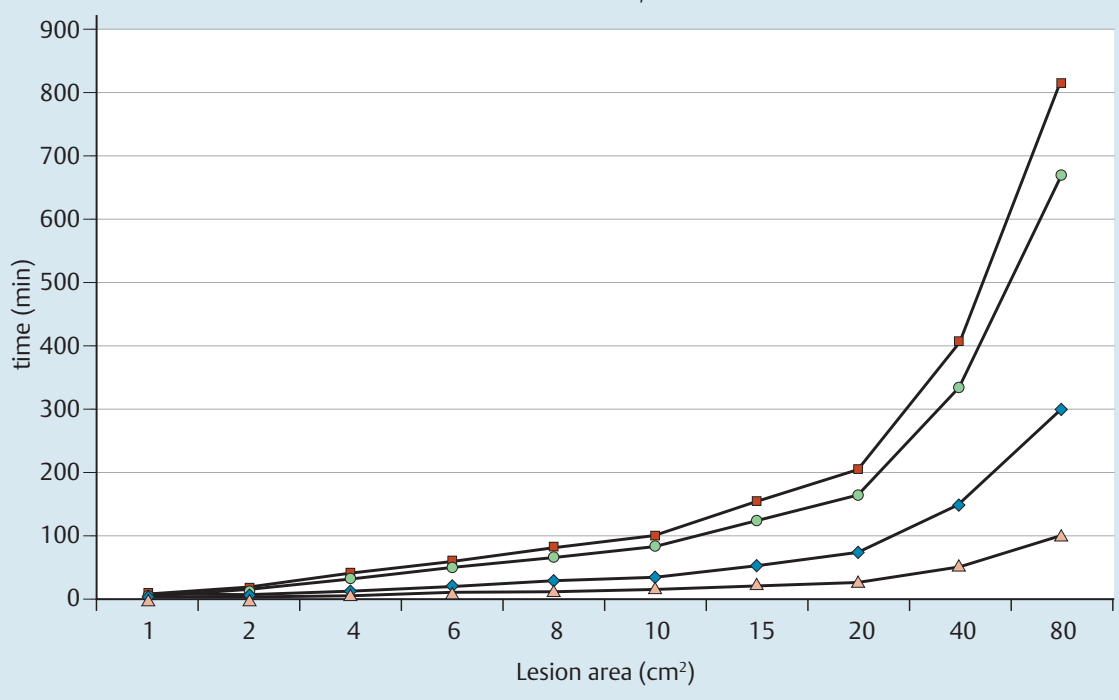

Regular ESD device min $\square$ Regular ESD device max $\triangle$ Nes ESD device min $\bigcirc$ Nes ESD device max
Fig.4 Time/surface ratio for completing and ESD with the standard ESD knife vs the new device. a Overall results in all animals. b Results for the submucosal phase of ESD in the second series of 4 pigs.
Fig. 5 A model of time/surface required to complete an ESD with a standard device vs the new device. Min and max are based on data observed during the animal study. saving, and the other two exhibiting a remarkable fall in sub-procedure time ( $\bullet$ Fig. 4 b). Finally, a simulation of the potential time saving as a function of the lesion area, based on observed minimum and maximum procedure time/area ratios with both devices, showed that the new device could save up to $66 \%$ of the procedure time when comparing minimum time/area ratios for both devices ( $\bullet$ Fig. 5).

\section{Discussion}

The new device evaluated in this study is similar to existing ESD knives in that it presents as a catheter-based, handle-controlled monopolar electrosurgical device. More specifically, it shares with the Flush-Knife ${ }^{\mathrm{TM}}$ a sideport for intraprocedural flushing to help clean the device tip and the operating field. However, this new ESD device differs from conventional ones in its unique versatility and the extended range of cutting wire to be put in contact with tissue, from the very tip for marking and incision to 
nearly a half snare for quick submucosal cut. As shown in the figures and videos, this ESD instrument can be used as a standard needle, as a hook that can be easily oriented to grasp and cut submucosal collagen fibers, and more specifically as a "scythe" with lateral motion of the endoscope or by using gentle forward motion of the wire fully extended and oriented in a plane parallel to that of the muscularis propria.

It is worth observing that in several experiments, ESD was achieved more expeditiously with the new device than with conventional ones to which both operators were used before starting the study, and that this time saving was entirely due to improved performance in the second part of the study, exhibiting a learning-curve effect. Although a trend to a decreased operating time over the course of the study was also visible with the regular knife, probably as a consequence of the standardization of lesion size and location ( $\boldsymbol{O}$ Fig. $\mathbf{4 a}$ ), this trend was more pronounced with the new knife despite having little or no experience with it, in contrast to the Flush-knife ${ }^{\mathrm{TM}}$. This learning curve seems relatively short, since fewer than five procedures were sufficient to observe some time savings that, while not statistically significant, was not detrimental to the safety of procedures. Previous similar studies of new devices failed to show a significant time-saving compared to earlier-generation ESD knives while exhibiting suboptimal versatility and possibly less accurate submucosal dissection [8]. Intra-procedural complications were a matter of concern because the device offers a long extension of bare wire that could harm and puncture the muscular layer. However, no complications were observed in either group and there were no more superficial muscular bruises with the new device ESDs than when the conventional device was used, on either macroscopic or microscopic examination. This is probably explained, in part, by the protective effect of the transparent hood.

A caveat for the use of the device in its "scythe" position is probably to avoid extending the catheter more than a few millimeters beyond the tip of the hood, as was done in this study. With that in mind, we consider the scythe shape to be the most innovative feature of this instrument, and the one that can save the most time in the submucosa, as observed in the swiftest of our procedures. It is difficult to know if the device presents a safety profile different from regular devices with regard to risk of bleeding because the pig model is not a good one for intraoperative bleeding, but there is no particular reason for concern on that issue. The new device has the same ability to cut a vessel and trigger bleeding and to coagulate it as do other knives. As with any other ESD knife, it is certainly necessary to consider having coagulation graspers at one's disposal when undertaking an ESD.

Another point this study cannot address completely is the ability of the device to achieve ESD in especially difficult locations. Because our goal was to perform two ESDs in reproducible conditions, we had to choose relatively "standard" locations and levels of difficulty. For example, we did not perform ESDs in retroflexed positions or in the distal part of the stomach, which in the pig model requires deep insertion of the endoscope with a long loop formation. We assume that the device's ability to conform to many types of angulation and lengths of active wire would make dissection much easier in such difficult locations than with other knives, but we recognize the need to confirm that with further studies. We wondered whether the flushing lumen could inject fluid with pressure sufficient to lift the submucosa during ESD. As indicated previously, we found that function to be suboptimal, but significant improvement has been made in that regard in more recent prototypes, which were not tested in this study. The last caveat for use of this new device is that some training is necessary to correctly manipulate the handle because it is slightly more complex than the push-pull handling of a standard knife or resection snare. Therefore, we recommend that a trained and skilled nurse be present when ESD is performed. Improvements to the handle are under way.

Finally, this study has several limitations: 1. Gastric ESD in the pig differs from that in humans because of the pig's distinctive gastric wall features (thick mucosa, few submucosal vessels, mucosal lifting easily sustained); 2.A small number of procedures were performed; and 3.Experience with the new device is limited. We consider this new device as of potentially significant help in making ESD both easier and less time-consuming without compromising the safety of procedures. Thus we are contemplating initiating a clinical study with this novel ESD knife.

Competing interests: The author (Frederic Prat) declare that a patent is pending with regard to the device investigated with coownership of Medwork GmbH and Assistance Publique-Hopitaux de Paris (AP-HP).

\section{Acknowledgment \\ $\nabla$}

The authors of this paper wish to thank the team of the Ecole de Chirurgie des Hôpitaux de Paris for their efficient support throughout this study.

\section{References}

1 Hosokawa K, Yoshida S. Recent advances in endoscopic mucosal resection for early gastric cancer. Gan To Kagaku Ryoho 1998; 25: 476-483

2 Yamamoto $H$, Yahagi $N$, Oyama $T$. Mucosectomy in the colon with endoscopic submucosal dissection. Endoscopy 2005; 37 : 764-768

3 Gotoda T, Yamamoto H, Soetikno RM. Endoscopic submucosal dissection of early gastric cancer. J Gastroenterol 2006; 41: $929-942$

4 Deprez PH, Bergman JJ, Meisner S et al. Current practice with endoscopic submucosal dissection in Europe: position statement from a panel of experts. Endoscopy 2010; 42: 853-858

5 Farhat S, Chaussade S, Ponchon $T$ et al. Endoscopic submucosal dissection in a European setting. A multi-institutional report of a technique in development. Endoscopy 2011; 43: 664-670

6 Kakushima N, Fujishiro M, Kodashima S et al. A learning curve for endoscopic submucosal dissection of gastric epithelial neoplasms. Endoscopy 2006; 38: 991 - 995

7 Lee WS, Cho JW, Kim YD et al. Technical issues and new devices of ESD of early gastric cancer. World J Gastroenterol 2011; 17: 3585 - 3590

8 Von Renteln D, Pohl H, Vassiliou MC et al. Endoscopic submucosal dissection by using a flexible Maryland dissector: a randomized, controlled, porcine study (with videos). Gastrointest Endosc 2010; 71: 1056- 1062

9 Neuhaus H, Wirths K, Schenk M et al. (2009) Randomized controlled study of EMR versus endoscopic submucosal dissection with a waterjet hybrid-knife of esophageal lesions in a porcine model. Gastrointest Endosc 2009; 70: $112-120$ 